\title{
The Hough transform and the impact of chronic leukemia on the compact bone tissue from CT-images analysis
}

\author{
Anna Maria Massone, Cristina Campi, Francesco Fiz, and Mauro Carlo Beltrametti
}

\begin{abstract}
Computational analysis of X-ray Computed Tomography (CT) images allows the assessment of alteration of bone structure in adult patients with Advanced Chronic Lymphocytic Leukemia (ACLL), and may even offer a powerful tool to assess the development of the disease (prognostic potential). The crucial requirement for this kind of analysis is the application of a pattern recognition method able to accurately segment the intra-bone space in clinical CT images of the human skeleton. Our purpose is to show how this task can be accomplished by a procedure based on the use of the Hough transform technique for special families of algebraic curves. The dataset used for this study is composed of sixteen subjects including eight control subjects, one ACLL survivor, and seven ACLL victims. We apply the Hough transform approach to the set of CT images of appendicular bones for detecting the compact and trabecular bone contours by using ellipses, and we use the computed semi-axes values to infer information on bone alterations in the population affected by ACLL. The effectiveness of this method is proved against ground truth comparison. We show that features depending on the semi-axes values detect a statistically significant difference between the class of control subjects plus the ACLL survivor and the class of ACLL victims.
\end{abstract}

Anna Maria Massone

Dipartimento di Matematica, Università di Genova, via Dodecaneso 35 - 16146 Genova, Italy, email: massone@dima.unige.it

Cristina Campi

Dipartimento di Medicina, Università di Padova, via Giustiniani 2 - 35128 Padova, Italy, e-mail: cristina.campi@unipd.it

Francesco Fiz

University of Tuebingen, Nuclear Medicine Unit, Department of Radiology, Hoppe-Seyler-Straße 3, 72076, Tübingen, Germany, e-mail: francesco.fiz.nm@gmail.com

Mauro Carlo Beltrametti

Dipartimento di Matematica, Università di Genova, via Dodecaneso 3516146 Genova, Italy, email: beltrametti@dima.unige.it 
Key words: X-ray tomography, image processing, pattern recognition, Hough transform, algebraic plane curves

\section{Introduction}

A computational analysis of a dataset of X-ray Computed Tomography (CT) images recently assessed the presence of alteration of bone structure in adult patients affected by Advanced Chronic Lymphocytic Leukemia (ACLL) [15]. These data (22 ACLL patients and 22 control subjects) have been analyzed by using a dedicated software, based on the use of active contour models [12, 28], to first identify the skeletal border from CT images, and then to segment regions corresponding to trabecular and compact bone (i.e., the two types of osseous tissue that form skeletal bones). The results showed that the whole body skeletal volume is similar in leukemic patients and in control subjects, while ACLL is associated with a significant trabecular bone volume enlargement, which prevails within the appendicular bones, this suggesting that leukemia causes a measurable bone erosion in the appendicular intraosseous space. Further, the degree of skeletal structure alteration displayed a relevant prognostic significance. For these reasons, the assessment of skeletal alterations of compact bone caused by ACLL might be used as a prognostic marker for the prediction of the clinical course of the disease.

The main limitation of the study presented in [15] is two-fold. On the one hand, the segmentation method based on active contours is utilized there just for the identification of the bone outer profile, while, for the recognition of the inner profile, a significantly less reliable heuristic approach based on thresholding is applied. On the other hand, only the bare volume values of the trabecular and compact bone have been considered, without taking into account geometrical aspects of the erosion.

Bone segmentation is an important task in biomedical imaging, and active contours have been widely used as reliable image segmentation methods. The fundamental idea in active contour models is to start with initial closed shapes, i.e., contours, and iteratively change them by applying shrink/expansion operations subject to constraints from a given image. The contour evolution is controlled by the minimization of an energy function. Truc et al. [30] applied several models toward bone segmentation of CT images. Among them, Gradient Vector Flow active contours [32], geometric active contours [20,33], geodesic active contours [11], Gradient Vector Flow Fast Geometric active contours [22], and Chan-Vese multi-phase active contours without edges [31] have been tested to segment knee bones from CT images. Constructing a graph from an image, the segmentation problem can be alternatively solved by using techniques for graph cuts in graph theory, where a graph cut is the process of partitioning a graph into disjoint sets. Graph cut framework for object segmentation was proposed in [7] and then developed into a large number of extensions based on either iterative parameter re-estimation and learning, multiscale or hierarchical approaches, and other techniques with a wide range of applications, including medical applications. An exhaustive survey of these developments 
is given in [6], together with different examples including segmentation of liver and lung lobes in CT volumes. Graph cuts are also used for the segmentation of vertebral bones from volumetric CT images [1]. Since bone structures are characterized by high intensity levels in CT images, their segmentation can also be obtained by using thresholding-based methods, where either a global or local thresholding approach can be followed. An example of this type of techniques is a fully automatic 3D adaptive thresholding method [34] that was proposed and tested on CT images of the calcaneus and vertebrae.

In the present paper we apply a pattern recognition method, based on a recent extension of the Hough Transform (HT) concept, able to detect both the trabecular and compact bone contours in CT images. When first introduced, the Hough transform technique was used to detect straight lines in images [17]. It is based on the pointline duality as follows: points in a straight line, defined by a linear equation in the image plane $\langle x, y\rangle$ with the usual natural parametrization, correspond to lines in the parameter space $\langle A, B\rangle$ that intersect in a single point. This point uniquely identifies the coefficients in the equation of the original straight line (analogous procedures to detect circles and ellipses in images have been then introduced in [14]). Further generalizations include Bayesian and fuzzy approaches to the Hough transform $[5,23]$, in addition to the well known generalized Hough transform [2], which allows the recognition of arbitrary shapes (even composite shapes like cars) by means of pre-set look-up tables (instead of analytic equations) where scale changes, rotations, figure-ground reversals, and reference point translation describing the shape of interest can be accounted for.

Recently, algebraic geometry arguments have been proposed in [4] in order to utilize the HT framework for special families of irreducible algebraic plane curves that share the degree, with applications to medical and astronomical images [21]. A further generalization proposes an iterative approach to the Hough transform technique for piecewise recognition of rather complex anatomical profiles [24].

This paper has two main objectives. The first objective is to evaluate the HT performances in quantifying the trabecular bone volume with respect to the previously employed technique [15]. To do this both techniques are compared with a ground truth given by manual segmentation from expert users. Our second objective is then to infer geometrical information regarding skeletal structure alterations from the recognized curve parameters and show that the set of parameters characterizing the detected curves can effectively provide prognostic information for ACLL. To achieve these goals, and following anatomical considerations, we consider families of ellipses to recognize appendicular bone contours. In particular, in order to study the bone erosion signature, we use as features of interest the ellipses semi-axes.

This paper is organized as follows. In Section 2, theoretical and computational details concerning the Hough transform technique, together with a description of the two families of curves here utilized, are presented. In Section 3, we provide some information concerning the patient recruitment and image acquisition details. Section 4 is devoted to achieve the first objective of the paper. Here we show how to address some preprocessing steps through an illustrative example, we apply the recognition technique to the detection of appendicular bone contours, and we offer a quantita- 
tive comparison of both, the proposed technique and the one previously employed, with a ground truth. In Section 5, we investigate the prognostic significance of the geometrical information inferred by using the HT technique from CT-images for the assessment of bone erosion due to ACLL, second objective of the paper. A brief discussion, together with our conclusions are then offered in Section 6. Finally, it is worth noticing that all the tests and analyses presented in this paper were performed within the Matlab computing environment.

\section{Background material}

In this section we recall some basic concepts concerning the Hough transform in the case of algebraic plane curves, and we describe two families of curves used in the recognitions presented in Section 4. We remark that the general framework here recalled can be exploited for the recognition tasks by using Hough regular families of algebraic curves defined below, including but not limited to straight lines, circles and ellipses. We refer to the first four sections of [25] for a complete, unified exposition on the Hough transform technique with respect to families of curves.

\subsection{Hough transform}

We follow the notation introduced in [4] and [21]. Let us consider a family of nonconstant irreducible real polynomials

$$
F(X, Y ; \lambda)=\sum_{i, j=0}^{d} g_{i j}(\lambda) X^{i} Y^{j}, \quad 0 \leq i+j \leq d,
$$

in the variables $X, Y$, where the coefficients $g_{i j}(\lambda)$ are evaluations in the independent parameters $\lambda=\left(\lambda_{1}, \ldots, \lambda_{t}\right)$, varying in an Euclidean open set $\mathcal{U} \subseteq \mathbb{R}^{t}$, of real polynomials $g_{i j}(\Lambda)$ in the variables $\Lambda=\left(\Lambda_{1}, \ldots, \Lambda_{t}\right)$. We assume that the degree of the polynomials $F(X, Y ; \lambda)$ does not depend on $\lambda$. Let $\mathcal{F}$ be the corresponding family of zero loci $C_{\lambda}$ of $F(X, Y ; \lambda)$, and assume that each $C_{\lambda}$ is an irreducible real curve in the affine plane $\mathbb{A}_{(X, Y)}^{2}(\mathbb{R})$, i.e., $C_{\lambda}$ is an irreducible curve over the complex field with infinitely many real points in $\mathbb{A}_{(X, Y)}^{2}(\mathbb{R})$. So we want a family $\mathcal{F}=\left\{C_{\lambda}\right\}$ of irreducible real curves (up to a finite number of isolated points) which share the degree.

If $P=\left(x_{P}, y_{P}\right)$ is a point of $\mathbb{A}_{(X, Y)}^{2}(\mathbb{R})$, then the Hough transform of $P$ (with respect to the family $\mathcal{F}$ ) is the algebraic locus $\Gamma_{P}(\mathcal{F})$ of the affine space $\mathbb{A}_{\left(\Lambda_{1}, \ldots, \Lambda_{t}\right)}^{t}(\mathbb{R})$ defined by the equation $\Gamma_{P}(\Lambda):=F\left(x_{P}, y_{P} ; \Lambda\right)=0$, where 


$$
F\left(x_{P}, y_{P} ; \Lambda\right)=\sum_{i, j=0}^{d} g_{i j}(\Lambda) x_{P}^{i} y_{P}^{j}, \quad 0 \leq i+j \leq d
$$

is a real polynomial in the indeterminates $\Lambda=\left(\Lambda_{1}, \ldots, \Lambda_{t}\right)$. For a general point $P$ in the image space, $\Gamma_{P}(\mathcal{F})$ is in fact a hypersurface, that is, a $(t-1)$-dimensional locus in the parameter space [26].

The following general facts hold true, as proved in $[4,25]$.

(a) The Hough transforms $\Gamma_{P}(\mathcal{F})$, when $P$ varies on $C_{\lambda}$, all pass through the point $\lambda$.

(b) Assume that the Hough transforms $\Gamma_{P}(\mathcal{F})$, when $P$ varies on $C_{\lambda}$, have a point in common other than $\lambda$, say $\lambda^{\prime}$. Thus the two curves $C_{\lambda}, C_{\lambda^{\prime}}$ coincide.

(c) (Regularity property) The following conditions are equivalent:

(i) for all curves $C_{\lambda}, C_{\lambda^{\prime}}$ in $\mathcal{F}$, the equality $C_{\lambda}=C_{\lambda^{\prime}}$ implies $\lambda=\lambda^{\prime}$;

(ii) for each curve $C_{\lambda}$ in $\mathcal{F}$, one has

$$
\bigcap_{P \in C_{\lambda}} \Gamma_{P}(\mathcal{F})=\lambda
$$

A family $\mathcal{F}$ which meets one of the above equivalent conditions is said to be Hough regular.

Condition (c-ii) is easy to be translated into a discrete framework for curves recognition in images: provided that an edge detection process selects in the image a set of points of interest potentially lying on the curve to be recognized, the intersection of their HTs leads to the identification of the parameter set characterizing the curve. Thus, we look for families $\mathcal{F}$ of curves which satisfy the above equivalent conditions. Condition (c-i) provides an effective way to check condition (c-ii). In fact, the equality $C_{\lambda}=C_{\lambda^{\prime}}$ is equivalent to $F(X, Y ; \lambda)=k F\left(X, Y ; \lambda^{\prime}\right)$ for some non-zero constant $k$. This leads to solve a polynomial system, in the variables $\lambda=\left(\lambda_{1}, \ldots, \lambda_{t}\right), \lambda^{\prime}=\left(\lambda_{1}^{\prime}, \ldots, \lambda_{t}^{\prime}\right)$, made up of the equations $g_{i j}(\lambda)=k g_{i j}\left(\lambda^{\prime}\right)$ for each pair of indices $i, j$ [4]. Therefore, saying that the family $\mathcal{F}$ is Hough regular simply means that such a polynomial system implies $\lambda=\lambda^{\prime}$.

Based upon the above theoretical result, a recognition algorithm can be implemented as follows. In short, first we apply to the image an edge detection technique to select $v$ points of interest, $P_{1}, \ldots, P_{v}$ (see Subsection 4.1 for a detailed description concerning how we deal with this step in the paper). Then, we discretize the parameter space by means of an appropriate number of cells and, for each point of interest $P_{j}, j=1, \ldots, v$, we compute the Hough transform $\Gamma_{P_{j}}(\mathcal{F})$ with respect to a fixed family $\mathcal{F}$ of curves. Next, we apply an accumulator function to count how many times each cell in the parameter space is crossed (voted) by the computed HTs. Finally, we look for the cell corresponding to the maximum of the accumulator function: the parameter set associated to that cell provides the curve of the family which best approximates the profile of interest in the image. 
The recognition algorithm

- Choose a set of points of interest, say $P_{j}, j=1, \ldots, v$, in the image space by applying an edge detection algorithm

- Consider a discretization in a region $\mathcal{T}$ of the parameter space given by the choice:

- sampling points $\lambda_{\mathbf{n}}=\left(\lambda_{1, n_{1}}, \lambda_{2, n_{2}}, \ldots, \lambda_{t, n_{t}}\right)$

- cells $\mathbf{C}_{\mathbf{n}}:=\left\{\Lambda \in \mathcal{T} \mid \lambda_{k} \in\left[\lambda_{k, n_{k}}-\frac{d_{k}}{2}, \lambda_{k, n_{k}}+\frac{d_{k}}{2}\right), k=1, \ldots, t, n_{k}=\right.$ $\left.1, \ldots, N_{k}\right\}$,

where $\mathbf{n}$ denotes the multi-index $\left(n_{1}, n_{2}, \ldots, n_{t}\right), d_{k}$ the sampling distance and $N_{k}$ the number of samples with respect to the component $k$

- Define an accumulator matrix $H=\left(H_{\mathbf{n}}\right)$

$$
H_{\mathbf{n}}=H_{n_{1}, n_{2}, \cdots, n_{t}}:=\#\left\{P_{j} \mid \Gamma_{P_{j}}(\mathcal{F}) \cap \mathbf{C}_{\mathbf{n}} \neq \emptyset, 1 \leq j \leq v\right\}
$$

- Optimize $H$

$$
\mathbf{n}^{*}:=\operatorname{argmax}_{\mathbf{n}} H_{\mathbf{n}}
$$

- Identify the set of optimal parameters $\lambda^{*}:=\lambda_{\mathbf{n}^{*}}=\left(\lambda_{1, n_{1}^{*}}, \lambda_{2, n_{2}^{*}}, \ldots, \lambda_{t, n_{t}^{*}}\right)$

- Characterize the equation of the seeked curve $C_{\lambda^{*}}$

\section{$>$ Remark I}

The computation of the accumulator function and its maximization is the most time-consuming step of the algorithm. Further, it strongly depends on the number of parameters, since the dimension of the domain of this function exactly corresponds to the number of parameters into play. Even though the theory, and the algorithmic aspects, presented in this section hold true in the above general framework, in practice, the computational burden associated to the accumulator function computation and optimization leads to the need of restricting to families of curves depending on a small number of parameters. On the other hand, evidence shows how to be able to control roto-translations and even scaling the variables is a matter of importance; see, for instance, the discussion in Subsection 2.2.2 below. In short, one should be able to study a family of curves of equation (1) up to coordinates transformations of type $(X, Y) \mapsto\left(s_{X} X+\mu_{1} s_{Y} Y+c_{1}, s_{Y} Y+\mu_{2} s_{X} X+c_{2}\right)$, where $s_{X}, s_{Y}$ are the scaling factors and $\mu_{1}, \mu_{2}, c_{1}, c_{2}$ take care of the roto-translation of the $X, Y$ axes, respectively. This increasing by up to six the number $t$ of the parameters $\lambda=\left(\lambda_{1}, \ldots, \lambda_{t}\right)$, and then making heavier all computations. Work to establish such a relevant extent is in progress. We also refer to [29] for related results. 


\section{Remark II}

The robustness of the recognition algorithm in presence of noise has been widely tested in [4], where the Hough transform algorithm showed to be extremely effective in recognizing curves when embedded in a very noisy framework (up to $99 \%$ of noise points), and against random perturbations of the location of the points on the curve.

Further, a bound for the number of points of interest to be considered in the curve, i.e. a bound for the number of Hough transforms to be considered for a successful optimization of the accumulator function in the recognition algorithm, is provided in [3]. Such a bound is consequence of geometrical arguments.

\subsection{Families of curves of interest}

We describe two families of curves. The first one is an illustrative example which also shows the extent of the HT framework in detecting curves in images, while the second one plays a crucial role in the paper.

\subsubsection{Curve of Lamet.}

Consider the family $\mathcal{F}=\left\{C_{a, b}\right\}$ of curves of degree $m$ of equation $\frac{X^{m}}{a^{m}}+\frac{Y^{m}}{b}=1$ for positive real numbers $a$ and $b$, or, in polynomial form (1),

$$
C_{a, b}: b X^{m}+a^{m} Y^{m}=a^{m} b .
$$

Clearly, the curve $C_{a, b}$ is non-singular. The curve of Lamet is bounded for even values of $m$. Note that, for instance, the case $m=3$ leads to the unbounded Fermat cubic curve. Indeed (see Example 8 in [25]) the curve of Lamet is contained in the rectangular region

$$
\left\{(x, y) \in \mathbb{A}_{(X, Y)}^{2}(\mathbb{R}) \mid-a \leq x \leq a,-b^{\frac{1}{m}} \leq y \leq b^{\frac{1}{m}}\right\} .
$$

For each point $P=\left(x_{P}, y_{P}\right)$ in the image plane, the HT is the $(m+1)$-degree curve in the parameter plane $\mathbb{A}_{(A, B)}^{2}(\mathbb{R})$ of equation

$$
\Gamma_{P}(A, B): B x_{P}^{m}+A^{m} y_{P}^{m}=A^{m} B .
$$

Let us assume now $C_{a, b}=C_{a^{\prime}, b^{\prime}}$. The regularity conditions $g_{i j}(\lambda)=k g_{i j}\left(\lambda^{\prime}\right)$, $(i, j) \in\{(m, 0),(0, m),(0,0)\}$, mentioned before, read in this case $b=k b^{\prime}, \quad a^{m}=$ $k a^{\prime m},-a^{m} b=-k a^{\prime m} b^{\prime}$ for some $k \in \mathbb{R} \backslash\{0\}$, respectively. Then $k a^{\prime m} b^{\prime}=a^{m} b=$ $k^{2} a^{\prime m} b^{\prime}$, so that $k^{2}=k$, whence $k=1$. Therefore $b=b^{\prime}$ and $a^{m}=a^{\prime m}$; since $a>0$ it follows $a=a^{\prime}$. Thus, the family $\mathcal{F}$ is Hough regular. 

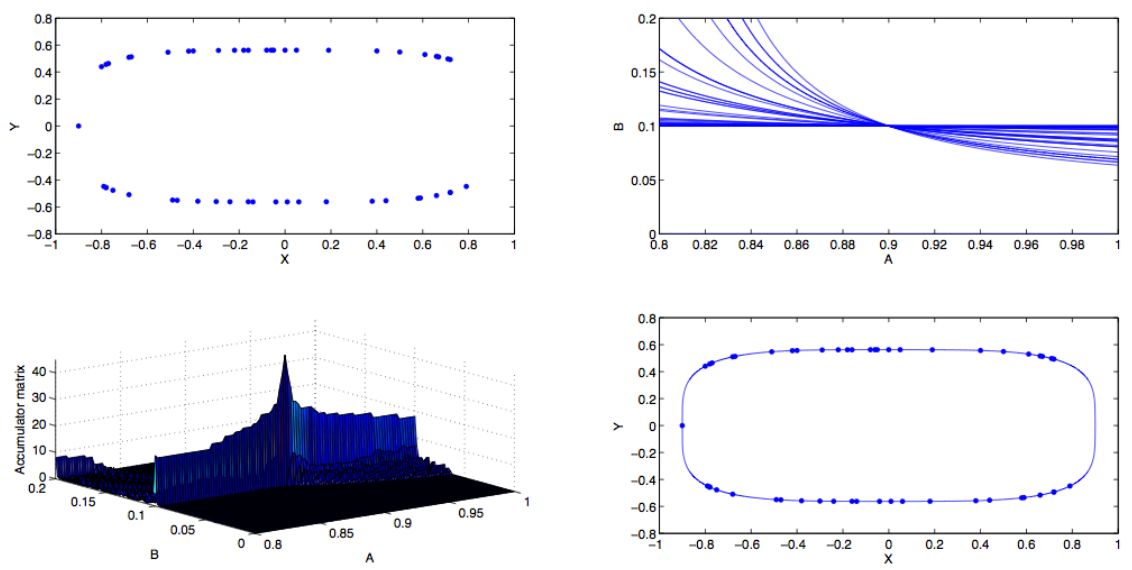

Fig. 1 Recognition of the curve of Lamet, equation (3) with $a=0.9, b=0.1, m=4$. Top left panel: dataset of points randomly sampled on the curve. Top right panel: Hough transforms of the dataset points. Bottom left panel: accumulator matrix. Bottom right panel: recognized curve with dataset points superimposed.

Finally, in [3] the optimal bound for the number of Hough transforms to be considered for a successful optimization of the accumulator function is proved to be $v_{o p t}=m^{2}+1$.

The effectiveness of the recognition algorithm in the case of the curve of Lamet with $m$ fixed to 4 is presented in Figure 1. For this illustrative example we construct a synthetic database of 50 points $\left(v_{o p t}=17\right)$ satisfying the curve equation (3) with $a=0.9$ and $b=0.1$ (see Figure 1, top left panel). Then the Hough transforms corresponding to all points of the database in the image space are expressed by equation (4) and drawn in the top right panel of the Figure. In the case we are considering, these transforms are 5-degree curves in the parameter plane $\mathbb{A}_{(A, B)}^{2}(\mathbb{R})$ that all meet in one point. The accumulator matrix is presented in the bottom left panel. The maximum of this function is clearly visible and it is used in order to determine the parameter values that uniquely identify the curve of Lamet in the image space (Figure 1, bottom right panel).

\subsubsection{Ellipse.}

To play with ellipses up to roto-translations, it is convenient to consider a family of ellipses expressed in the more general form, as follows. First, look at the general conic of equation $\lambda_{0} X^{2}+\lambda_{1} X Y+\lambda_{2} Y^{2}+\lambda_{3} X+\lambda_{4} Y+\lambda_{5}=0$. To be an ellipse, we need $\lambda_{0} \lambda_{2} \neq 0$, and $\lambda_{1}^{2}-4 \lambda_{0} \lambda_{2}<0$. Thus, we can for instance assume $\lambda_{0}=1$ and look at the 5-parametrized family $\mathcal{F}=\left\{\mathcal{E}_{\lambda}\right\}$ of ellipses expressed in the form

$$
\mathcal{E}_{\lambda}: X^{2}+\lambda_{1} X Y+\lambda_{2} Y^{2}+\lambda_{3} X+\lambda_{4} Y+\lambda_{5}=0,
$$


where $\lambda_{1}^{2}<4 \lambda_{2}$ and det $M \neq 0, M$ being the coefficient matrix of equation (5). The region $\mathcal{U} \subset \mathbb{R}^{5}$ where the parameters $\lambda=\left(\lambda_{1}, \ldots, \lambda_{5}\right)$ vary is then defined by such conditions.

By using the standard invariance and reduction theorems [13], the equation of the ellipse $\mathcal{E}_{\lambda}$ reduces to the canonical form, with respect to a new system of coordinates $\left\langle O^{\prime}, X^{\prime}, Y^{\prime}\right\rangle$,

$$
\mathcal{E}_{a, b}: \frac{X^{\prime 2}}{a^{2}}+\frac{Y^{\prime 2}}{b^{2}}=1,
$$

where the semi-axes $a, b \in \mathbb{R}_{+}$are given by

$$
a=\left(-\frac{\operatorname{det} M}{t_{1}^{2} t_{2}}\right)^{1 / 2}, \quad b=\left(-\frac{\operatorname{det} M}{t_{1} t_{2}^{2}}\right)^{1 / 2},
$$

with $t_{1}, t_{2}$ the eigenvalues of the submatrix $M_{33}=\left(\begin{array}{cc}1 & \lambda_{1} / 2 \\ \lambda_{1} / 2 & \lambda_{2}\end{array}\right)$. The fact that the conic is an ellipse assures that

$$
-\operatorname{det} M /\left(t_{1}^{2} t_{2}\right)>0, \quad-\operatorname{det} M /\left(t_{1} t_{2}^{2}\right)>0 .
$$

Moreover, note that equation (6) is defined up to a rotation by an angle of $\pi / 2$, which is enough for our purposes.

A straightforward check shows that both the families $\left\{\mathcal{E}_{\lambda}\right\}$ and $\left\{\mathcal{E}_{a, b}\right\}$ are Hough regular.

For each point $P=\left(x_{P}, y_{P}\right)$ in the image plane, the HT of $P$, with respect to the family $\mathcal{F}$, is the hyperplane in the parameter space $\mathbb{A}_{\left(\Lambda_{1}, \ldots, \Lambda_{5}\right)}^{5}(\mathbb{R})$ of equation

$$
\Gamma_{P}(\Lambda): x_{P}^{2}+\Lambda_{1} x_{P} y_{P}+\Lambda_{2} y_{P}^{2}+\Lambda_{3} x_{P}+\Lambda_{4} y_{P}+\Lambda_{5}=0 .
$$

Finally, following [3], one has $v_{o p t}=d^{2}+1$, where $d$ is the degree of the curves of the family, so that $v_{o p t}=5$ for the ellipses.

\section{Patient recruitment and image acquisition}

The study presented in this paper is concerned with an analysis of CT-images for the recognition of bone contours of a subset including sixteen subjects among the ones analyzed in [15] (precisely, we have processed eight control subjects belonging to a published normalcy dataset [28], one ACLL survivor, and seven patients, which died because of ACLL). The study presents a retrospective analysis of imaging data, gathered for a valid clinical reason. This analysis was authorized by the Local Ethics Committee (Comitato Etico Regionale Liguria), and influenced in no way the clinical decision making. All patients signed an informed consent prior of study inclusion. Inclusion criteria, as well as imaging technique, have been previously described [15]. Briefly, the study included ACLL patients with no previous specific 
treatment and recent disease progression. Further exclusion criteria included clinical history of other solid or hematologic malignancy, previous prolonged corticosteroid therapy, previous or ongoing therapy with drugs affecting skeletal metabolism (such as bisphosphonates or denosumab), uncontrolled diabetes, active infection and recent use of erythropoietin, G-CSF or other BM-stimulating drugs. PET/CT imaging started one hour after bolus injection of 18F-fluorodeoxyglucose (FDG, 4.8-5.2 MBq per kilogram of body weight). The exam was performed in the threedimensional mode, from vertex to toes in an arms-down position, using an integrated PET/CT scanner (Hirez; Siemens Medical Solutions, Knoxville, Tennessee). PET raw data were reconstructed by means of Ordered Subset Expectation Maximization [18] (3 iterations, 16 subsets), and attenuation correction was performed by using CT data. The transaxial field of view and pixel size of the reconstructed PET images were $58.5 \mathrm{~cm}$ and $4.57 \mathrm{~mm}$, respectively, with a $128 \times 128$ matrix. A 16-detector row helical CT scan was performed with non-diagnostic current and voltage settings, with a gantry rotation speed of 0.5 second and a table speed of 24 $\mathrm{mm}$ per gantry rotation. No contrast medium was injected. The entire CT data set was fused with the three-dimensional PET images by using an integrated software interface (Syngo; Siemens, Erlangen, Germany).

\section{Image analysis}

The basic step of the analysis presented in this paper is the identification of bone contours in CT data, here performed by using an extended version of the Hough transform recognition algorithm $[17,14,4]$. The Hough transform is widely used in image processing to detect curves (whose equations depend on a set of parameters) in images. The basic idea of this recognition procedure is that points lying on a curve in the image space can be transformed into hypersurfaces (their Hough transforms) in the parameter space, and the set of parameters corresponding to the intersection of all Hough transforms identifies the curve to be recognized in the image space. As shown in Figure 1, from a computational point of view a histogram (the Hough accumulator) can be defined on the discretized parameter space: for each cell in the parameter space, the value of the accumulator corresponds to the number of Hough transforms passing through that cell. The position of the maximum in the Hough counter identifies the set of parameters characterizing the curve to be detected in the image space.

In order to show how this technique works in the context of this paper, in Subsection 4.1 we present an illustrative example concerning the identification of sternum profiles in the thoracic skeleton. Even though the thoracic skeleton is not included in the following analysis (bone erosion signature has to be found in the appendicular skeleton mainly), this example gives us a chance first, to show the capability of the HT-based algorithm in detecting a non trivial anatomical profile and second, to discuss crucial methodological issues like the selection in the image of the points to be HT-transformed. Then, in Subsection 4.2, we show in detail the way we pro- 

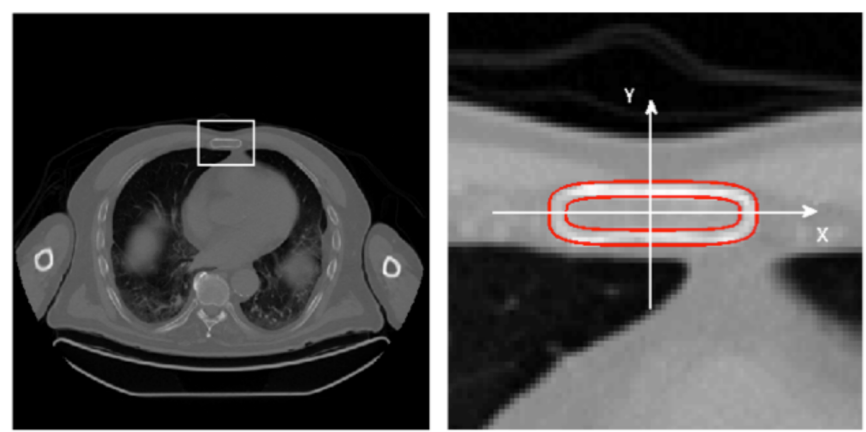

Fig. 2 Recognition of the inner and outer profiles of a sternum. Left panel: Original X-ray CT image with focus on the portion of interest. Right panel: Curves of Lamet (red lines), equation (3), providing the best approximation of the outer $(a=2.025, b=0.141)$ and inner $(a=1.670$, $b=0.011)$ sternum profiles superimposed to the original image.

cessed the data, and we describe the analyses and comparisons performed in order to evaluate the reliability of our results with respect to a ground truth given by manual segmentation from expert users.

\subsection{An illustrative example}

The sternum is one of the skeleton sites where the bone marrow is abundantly present. Bone marrow is the main hematopoietic tissue in adult humans and its examination can be used for the diagnosis of several diseases, like leukemia. For these reasons, it is important to distinguish between the compact and trabecular bone of this particular district. From a visual inspection of several CT images, it emerges that the sternum profile can be effectively described by using the curve of Lamet, introduced in Subsection 2.2.1 (see [8,9]). Then, the identification task is performed by means of the HT via curves of Lamet with $m$ fixed to an even value in order to deal with a bounded curve (see equation (3)). In particular, we chose the value $m=4$ proved to be effective in a previous work [8]. Left panel of Figure 2 shows an axial view of a CT image of a thorax. The white box in the image outlines the sternum region. The result of the identification of the inner and outer bone contours, i.e., the two curves of Lamet (solid lines) associated with the recognized parameters, is given in the right panel.

As a preprocessing step, Canny edge detection [10] (with parameters set to their Matlab default values) followed by a search for connected components [16] in the edge image (Figure 3, left panel) is used to select the image points of interest, i.e., the ones to be Hough-transformed (due to the anatomical conformation of the sternum, we have discarded all components having points at the borders of the image). The resulting set of points of interest is then shown in the right panel of Figure 3. A coordinate system (as the one outlined in the right panel of Figure 2) is then au- 


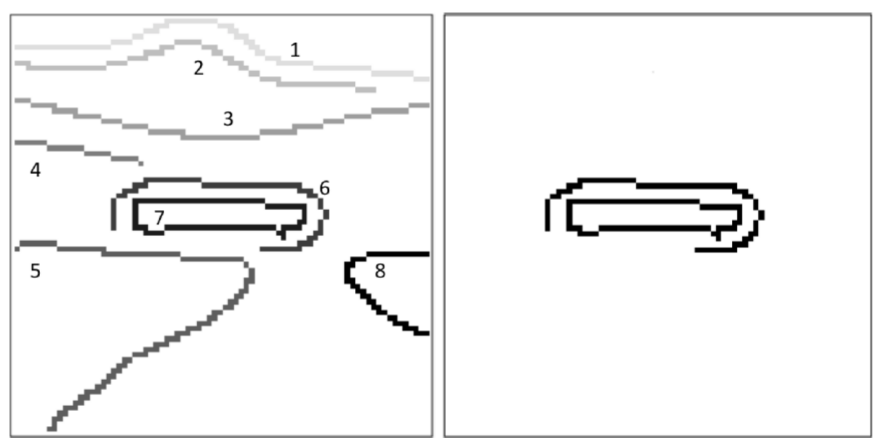

Fig. 3 Extraction of the sternum edge points. Left panel: Application of Canny edge detection to the portion of interest outlined in the left panel of Figure 2, and resulting identification of eight 8-connected components. Right panel: Rejection of some connected components and extraction of the set of points of interest to be Hough-transformed.

tomatically defined in order to have the origin coincident with the center of mass of these identified sternum edge points (the center of mass approximately corresponds to the middle of the trabecular region). No roto-translation with respect to this coordinate system was necessary here. It is worth noticing that the procedure described in this section for the points of interest extraction will be also used in the following subsection for the recognition of bone contours in the appendicular skeleton.

As a final remark we would like to point out the robustness of the method in recognizing the outer profile of the sternum even though a wide portion of edge points was not extracted by the edge detection algorithm.

\subsection{Analysis of the appendicular skeleton}

For each one of the sixteen subjects at disposal, the dataset consists of images from whole-body CT scanning $(512 \times 512$ pixels per slice, $1.36 \mathrm{~mm}$ size each pixel, and $5 \mathrm{~mm}$ thick images), the number of slices depending on the subjects' height and acquisition modality (for a minimum and maximum number of slices equal to 302 and 476, respectively). Among them we have considered a stack of CT-images corresponding to appendicular bones (i.e., femurs and humeri). For each subject, and for each appendicular bone, we have selected the first and last slice to process, together with a Region Of Interest (ROI) whose dimension was $60 \times 60$ pixels. The same ROI was then automatically replicated to each slice in between. Table 1 shows the minimum and maximum number of considered slices, for each appendicular bone, across the population of sixteen subjects.

We applied the Hough transform approach to the whole set of CT images of humeri and femurs for detecting the inner and outer bone contours by using ellipses as prototype curves (see Figures 4 and 5). To this aim, for each ROI containing either a femur or a humerus, we first selected the points of interest by following the same 
Table 1 Minimum and maximum number of slices, corresponding to each appendicular bone in a dataset of images from whole-body CT scanning of sixteen subjects differing for age and sex.

\begin{tabular}{lll}
\hline & Minimum & Maximum \\
\hline Left Humerus & 27 & 59 \\
Right humerus & 25 & 58 \\
Left femur & 38 & 90 \\
Right femur & 36 & 92 \\
\hline
\end{tabular}

edge detection-based procedure described in the previous subsection. In Table 2 we report the mean numbers of points of interest, with standard deviations, extracted from the Canny edge detection algorithm for both, the inner and outer bone profile, and for each appendicular bone. Recalling that, in the case of the ellipses, the optimal number of points of interest to guarantee a successful recognition is $v_{o p t}=5$, we can conclude that the cardinality of the datasets at disposal is high enough to guarantee reliable results for all the considered anatomical districts. We introduced a coordinate system centered in the center of mass of the extracted points of interest (i.e., in the trabecular tissue), and finally we used the 5-parametrized family of ellipses $\mathcal{E}_{\lambda}$ of equation (5), which automatically takes into account possible rototranslations. The optimal set of parameters $\lambda=\left(\lambda_{1}, \ldots, \lambda_{5}\right)$, i.e., the output of the recognition algorithm, was then used to compute the semi-axes values ( $a$ and $b$ ) by means of relations (7), from which it was also easy to compute the trabecular and compact bone areas (as $\pi a b$ ), and the intraosseous volume (by summing up the area values across different slices).

As for the computational time necessary to accomplish the whole process, we used the Matlab cputime code to evaluate the CPU time, in seconds, used to run the recognition algorithm on a $2.9 \mathrm{GHz}$ Intel Core i7 processor. Our results show that for a full analysis of a subject about 200 seconds are enough, 120 of which aimed at analysing the outer profiles of both, the right and left femurs.

Table 2 Mean numbers of points of interest, and corresponding standard deviation values, extracted by the edge detection step from the inner and outer bone contours, for each appendicular bone.

\begin{tabular}{lll}
\hline & Inner contour & Outer contour \\
\hline Left Humerus & $22 \pm 6$ & $47 \pm 10$ \\
Right humerus & $23 \pm 8$ & $48 \pm 10$ \\
Left femur & $34 \pm 11$ & $65 \pm 14$ \\
Right femur & $34 \pm 11$ & $65 \pm 14$ \\
\hline
\end{tabular}

\subsubsection{Comparison with ground truth}

As we pointed out in the Introduction, the main limitation of the study presented in [15] lies in the fact that the segmentation method based on active contours was 


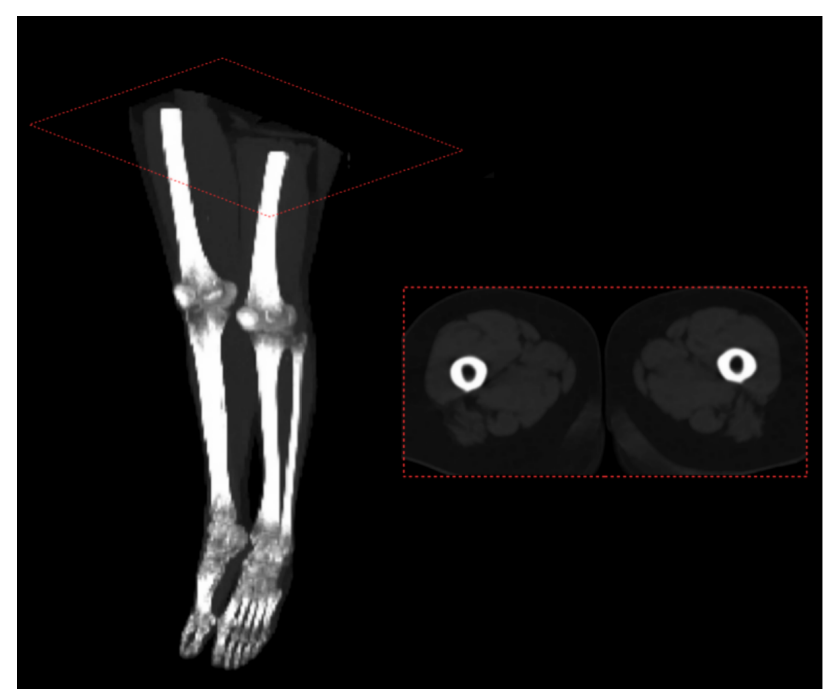

Fig. 4 Appendicular skeleton: 3D (left panel) and an axial view (right panel) of the leg bones. In the axial view the compact bone of the femurs (white areas) is clearly visible.

utilized just for the identification of the bone outer profile, while, for the recognition of the inner profile, a less reliable approach based on thresholding was applied. The HT-based approach overcomes this limitation since it is designed to automatically detect both the trabecular and compact bone contours. Taking advantage from this fact, the first aim of this paper is to investigate whether the use of a more accurate detection of the inner profile leads to more reliable results. We have then compared both, the results provided by the HT-based method, and the method based on ac-
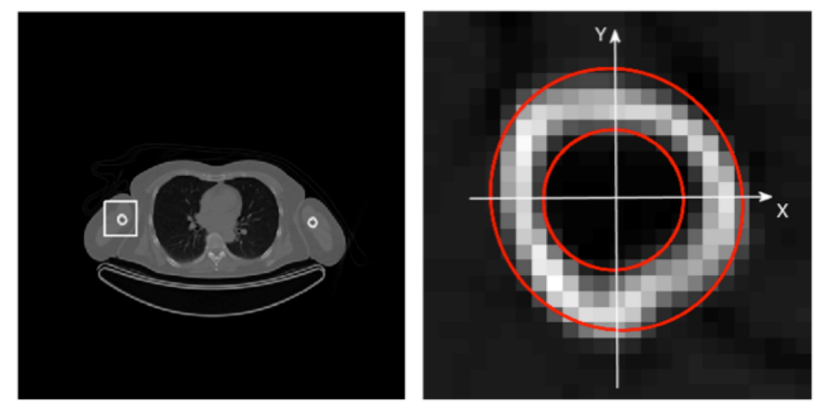

Fig. 5 Recognition of the inner and outer profiles of a humerus. Left panel: Original X-ray CT image with focus on the portion of interest. Right panel: Ellipses, of equation (5), providing the best approximation of the outer and inner profiles superimposed to the original image. Outer ellipse: $\lambda_{1}=-0.102, \lambda_{2}=0.941, \lambda_{3}=0.014, \lambda_{4}=0.146, \lambda_{5}=-2.629$, values leading to $a=1.7$ and $b=1.6$ by using relations (7). Inner ellipse: $\lambda_{1}=0, \lambda_{2}=1, \lambda_{3}=0.098, \lambda_{4}=0.144$, $\lambda_{5}=-0.802$, values leading to $a=b=0.9$ by using relations (7). 

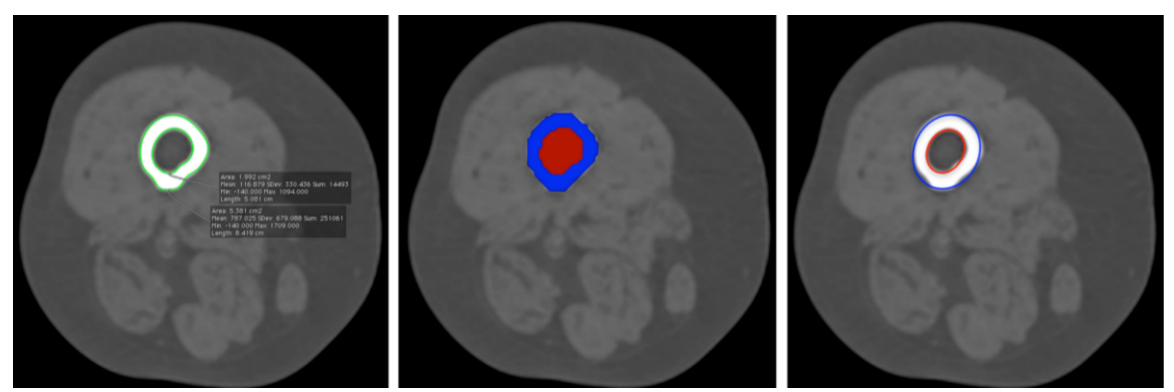

Fig. 6 Computation of the trabecular bone area $\left(A_{\text {in }}\right)$ and the whole bone area $\left(A_{\text {out }}\right)$ in a slice of a femur. Left panel: Ground truth, i.e., manually drawn profiles within the OsiriX software package $\left(A_{\text {in }}^{\mathrm{GT}}=1.99 \mathrm{~cm}^{2}, A_{\text {out }}^{\mathrm{GT}}=5.38 \mathrm{~cm}^{2}\right)$. Middle panel: Active contours/thresholding segmentation $\left(A_{\mathrm{in}}^{\mathrm{ACT}}=2.31 \mathrm{~cm}^{2}, A_{\mathrm{out}}^{\mathrm{ACT}}=5.48 \mathrm{~cm}^{2}\right)$. Right panel: Hough transform by using ellipses $\left(A_{\mathrm{in}}^{\mathrm{HT}}=\right.$ $\left.1.88 \mathrm{~cm}^{2}, A_{\text {out }}^{\mathrm{HT}}=5.43 \mathrm{~cm}^{2}\right)$.

tive contours/thresholding, with a ground truth given by manual segmentation from expert users. Specifically, for each subject, and for each slice of each appendicular bone, the internal and external profiles of the bone were drawn by hand using the OsiriX package [27]; the area within each profile was then automatically computed by the software (see left panel of Figure 6), and the values compared with the ones obtained by using the active contours/thresholding model (see middle panel of Figure 6) and the Hough transform (see right panel of Figure 6).

For each subject $s, s=1, \ldots, 16$, we introduce the following notation:

- $i \in\{1,2,3,4\}$, an index identifying the left and right humeri and the left and right femurs, in the order;

- $n(s, i)$, the number of images at disposal corresponding to the $i$-th appendicular bone, as labelled in the previous item;

- $j=1, \ldots, n(s, i)$, an index identifying the $j$-th image of the $i$-th appendicular bone;

- $A_{\mathrm{in}}^{\mathrm{GT}}(s, i, j), A_{\mathrm{in}}^{\mathrm{ACT}}(s, i, j)$, and $A_{\mathrm{in}}^{\mathrm{HT}}(s, i, j)$, the trabecular bone areas (i.e, inner areas) computed in the $j$-th image of the $i$-th appendicular bone, by using OsiriX (ground truth), the active contours/thresholding method, and the Hough transform technique, respectively;

- $A_{\text {out }}^{\mathrm{GT}}(s, i, j), A_{\text {out }}^{\mathrm{ACT}}(s, i, j)$, and $A_{\text {out }}^{\mathrm{HT}}(s, i, j)$, the whole (trabecular plus compact bone) areas (i.e., outer areas) computed in the $j$-th image of the $i$-th appendicular bone, by using OsiriX (ground truth), the active contours/thresholding method, and the Hough transform technique, respectively.

For each image, we computed the percentage error originated by the last two methods in the evaluation of the trabecular bone area with respect to the ground truth value:

$$
e r r_{\mathrm{in}}^{\mathrm{ACT}}(s, i, j)=\frac{A_{\mathrm{in}}^{\mathrm{ACT}}(s, i, j)-A_{\mathrm{in}}^{\mathrm{GT}}(s, i, j)}{A_{\mathrm{in}}^{\mathrm{GT}}(s, i, j)}
$$




$$
e r r_{\mathrm{in}}^{\mathrm{HT}}(s, i, j)=\frac{A_{\mathrm{in}}^{\mathrm{HT}}(s, i, j)-A_{\mathrm{in}}^{\mathrm{GT}}(s, i, j)}{A_{\mathrm{in}}^{\mathrm{GT}}(s, i, j)},
$$

and, analogously, for the whole bone areas:

$$
\begin{aligned}
e r r_{\mathrm{out}}^{\mathrm{ACT}}(s, i, j) & =\frac{A_{\mathrm{out}}^{\mathrm{ACT}}(s, i, j)-A_{\text {out }}^{\mathrm{GT}}(s, i, j)}{A_{\mathrm{out}}^{\mathrm{GT}}(s, i, j)} \\
e r r_{\mathrm{out}}^{\mathrm{HT}}(s, i, j) & =\frac{A_{\mathrm{out}}^{\mathrm{HT}}(s, i, j)-A_{\text {out }}^{\mathrm{GT}}(s, i, j)}{A_{\mathrm{out}}^{\mathrm{GT}}(s, i, j)} .
\end{aligned}
$$

Note that in relations (8)-(11), we intentionally avoided the use of the absolute values to account for possible underestimations or overestimations of the quantity into play. Further, the computed values can be analyzed in many different ways by averaging over different indices. Here we offer an overall comparison by defining, for each method used and with obvious meaning of the notations, the overall mean percentage errors as:

$$
\begin{aligned}
E r r_{\mathrm{in}}^{\mathrm{ACT}} & =\frac{1}{K} \sum_{s} \sum_{i} \frac{1}{n(s, i)} \sum_{j=1}^{n(s, i)} \operatorname{err}_{\mathrm{in}}^{\mathrm{ACT}}(s, i, j) \\
E r r_{\mathrm{out}}^{\mathrm{ACT}} & =\frac{1}{K} \sum_{s} \sum_{i} \frac{1}{n(s, i)} \sum_{j=1}^{n(s, i)} \operatorname{err}_{\mathrm{out}}^{\mathrm{ACT}}(s, i, j) \\
E r r_{\mathrm{in}}^{\mathrm{HT}} & =\frac{1}{K} \sum_{s} \sum_{i} \frac{1}{n(s, i)} \sum_{j=1}^{n(s, i)} \operatorname{err}_{\mathrm{in}}^{\mathrm{HT}}(s, i, j) \\
E r r_{\mathrm{out}}^{\mathrm{HT}} & =\frac{1}{K} \sum_{s} \sum_{i} \frac{1}{n(s, i)} \sum_{j=1}^{n(s, i)} \operatorname{err}_{\mathrm{out}}^{\mathrm{HT}}(s, i, j),
\end{aligned}
$$

where $K=16 \times 4$ is a constant allowing one to average over the total number of subjects and appendicular bones. The four computed values, together with the corresponding standard deviations, are summarized in Table 3, where we can appreciate how the active contours/thresholding method overestimates the trabecular bone volume with respect to the ground truth, coherently with a non-optimal performance of the thresholding step in the bone segmentation process. The other three values seem

Table 3 Overall mean percentage errors, and corresponding standard deviations, computed by using Equations (12-15).

\begin{tabular}{ll}
\hline Active contours/thresholding & Hough transform \\
\hline $\operatorname{Err}_{\text {in }}^{\mathrm{ACT}}=(0.1 \pm 0.2)$ & $\operatorname{Err}_{\text {in }}^{\mathrm{HT}}=(-0.06 \pm 0.09)$ \\
$\operatorname{Err}_{\mathrm{out}}^{\mathrm{ACT}}=(-0.1 \pm 0.1)$ & $\operatorname{Err}_{\mathrm{out}}^{\mathrm{HT}}=(-0.01 \pm 0.08)$ \\
\hline
\end{tabular}


to slightly underestimate the corresponding quantities. All values look very accurate within their standard deviations, even though the HT approach shows, as expected, a more precise behaviour with error values smaller of an order of magnitude than the active contours/thresholding approach. An analogous analysis performed by dividing the population in two classes (control subjects plus the ACLL survivor, ACLL victims) provided very similar results.

\section{Prognostic significance}

The results presented in [15], and briefly summarized in Section 1, show that the effects of ACLL on skeletal structure cause a significant expansion of the intraosseous volume (in particular in the appendicular skeleton) and no significant alterations in the skeletal bone volume, i.e., ACLL erodes the compact bone from within the trabecular tissue.

In our framework, this evidence translates in the fact that no significant alterations are expected on the semi-axes values associated to the outer ellipses, over the whole population. More importantly, in the high-risk population, alterations in the compact bone due to erosion must be mirrored in increases of the semi-axes values associated to the inner ellipses with respect to the corresponding values in control subjects. The second aim of this study was then to investigate whether geometrical information related to the inferred semi-axes values can provide prognostic information for ACLL. Specifically, by introducing the following notation:

- $a_{\text {in }}(s, i, j)$ and $b_{\text {in }}(s, i, j)$, the semi-axes of the inner ellipse recognized in the $j$-th image of the $i$-th appendicular bone for the $s$-th subject;

- $a_{\text {out }}(s, i, j)$ and $b_{\text {out }}(s, i, j)$, the semi-axes of the outer ellipse recognized in the $j$-th image of the $i$-th appendicular bone for the $s$-th subject,

we expect both the ratios $\frac{a_{\text {in }}}{a_{\text {out }}}$ and $\frac{b_{\text {in }}}{b_{\text {out }}}$ to be smaller in control subjects than in leukemic patients, as the indices $s, i$ and $j$ vary.

To investigate the geometrical structure of the femurs and humeri (and the corresponding alterations in the inner semi-axes values in case of disease), we defined the following features:

$$
\begin{aligned}
r_{a}(s, i) & =\frac{1}{n(s, i)} \sum_{j=1}^{n(s, i)} \frac{a_{\text {in }}(s, i, j)}{a_{\text {out }}(s, i, j)}, \\
r_{b}(s, i) & =\frac{1}{n(s, i)} \sum_{j=1}^{n(s, i)} \frac{b_{\text {in }}(s, i, j)}{b_{\text {out }}(s, i, j)}, \\
r_{a b}(s, i) & =\frac{1}{n(s, i)} \sum_{j=1}^{n(s, i)} \frac{a_{\text {in }}(s, i, j) b_{\text {in }}(s, i, j)}{a_{\text {out }}(s, i, j) b_{\text {out }}(s, i, j)},
\end{aligned}
$$


Fig. 7 Distribution of dead patients (black triangles), survived patient (red circle) and controls (green crosses) in terms of the values $R_{a}^{(s)}, R_{b}^{(s)}$ and $R_{a b}^{(s)}$ in $\mathbb{A}_{\left(R_{a}, R_{b}, R_{a b}\right)}^{3}(\mathbb{R})$,

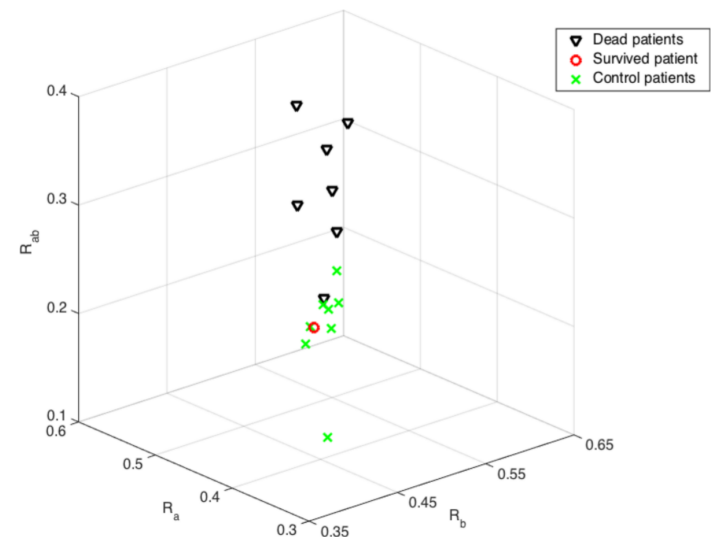

where, for the $s$-th subject and for the $i$-th appendicular bone, $r_{a}(s, i)$ represents the mean value of the ratio between the inner and outer value of the semi-axis $a$. Analogously, $r_{b}(s, i)$ represents the mean value of the ratio between the inner and outer value of the semi-axis $b$, and eventually $r_{a b}(s, i)$ represents the mean value of the ratio between the areas of the inner and outer ellipses.

Relations (16)-(18) provide information for each subject, and for each appendicular bone. We have processed these information in two different ways.

First, averaging each one of the previous mean ratios over the four anatomical districts (i.e., with respect to the index $i$ ) allowed us to identify each subject $s$ by a point $\left(R_{a}^{(s)}, R_{b}^{(s)}, R_{a b}^{(s)}\right) \in(0,1) \times(0,1) \times(0,1)$, that is, belonging to the space $\mathbb{A}_{\left(R_{a}, R_{b}, R_{a b}\right)}^{3}(\mathbb{R})$ (see Figure 7). In this feature space the high-risk subjects are expected to be the points closest to $(1,1,1)$. In Figure 7 we show the distribution of the sixteen subjects with respect to these three features: the survived patient (red circle) falls in the middle of the cloud of the control subjects (green crosses), while the dead patients (black triangles) show a more scattered behavior towards higher values of the three features, as expected. Impact of $R_{a}^{(s)}, R_{b}^{(s)}$ and $R_{a b}^{(s)}$ values on

Fig. 8 Kaplan-Meier curves for the two groups of subjects defined on the basis of the median value of their $R_{a}$ parameter values.

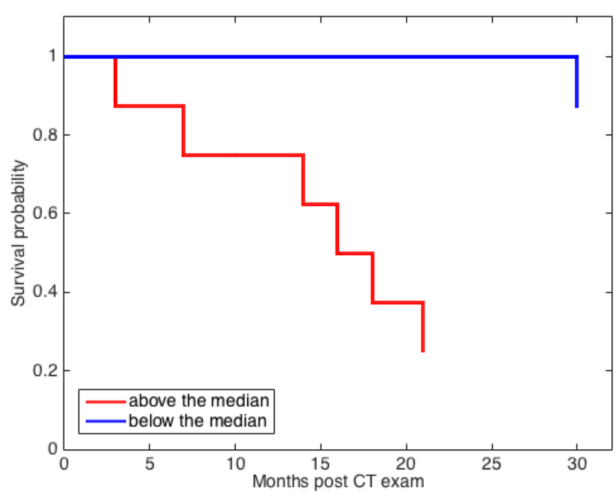



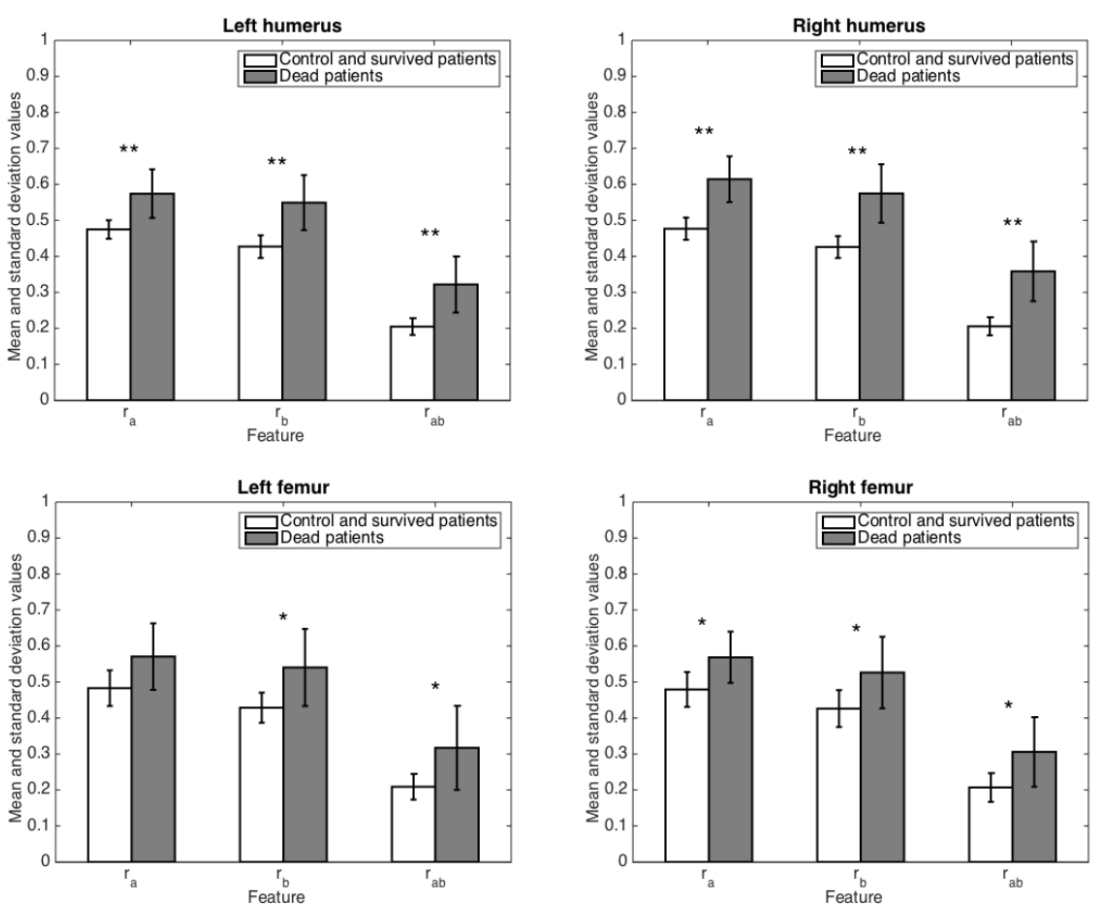

Fig. 9 Measure of the mean values $r_{a}(i), r_{b}(i), r_{a b}(i)$ and corresponding standard deviations for two populations: control subjects plus the ACLL survivor (white boxes) and ACLL victims (grey boxes). Left humerus $(i=1)$. Right humerus $(i=2)$. Left femurs $(i=3)$. Right femurs $(i=4)$. The significance levels $p$ (t-test) are also indicated $(* *:=p \leq 0.01$, and $*:=0.01<p \leq 0.05)$.

survival was tested with the Kaplan-Meier [19] analysis. For each parameter $R_{a}$, $R_{b}, R_{b}$, the population was stratified in two subgroups, using their median values as a discriminant. For instance, in the case of the $R_{a}$ parameter, the majority of events occurred in the subgroup with $R_{a}$ above the median; accordingly, two-years survival was $87.5 \%$ and $25 \%$ for the subgroups below and above the median, respectively. Figure 8 depicts the survival curve associated with the parameter $R_{a}$. Indeed, this analysis produced identical results for the parameters $R_{b}$ and $R_{a b}$ as well. These data suggest a possible prognostic role of the Hough transform based analysis, which appears to be able to identify a high-risk sub-population, recognized on the base of osseous alterations.

After dividing the population in two classes (control subjects plus the ACLL survivor, ACLL victims), a second way to process information given from relations (16)-(18) is to average the $r_{a}(s, i), r_{b}(s, i)$ and $r_{a b}(s, i)$ values over these two subpopulations (i.e., with respect to two disjoint subsets of indices $s$ ). In Figure 9, for each appendicular bone and for each sub-population, we show the resulting mean values, $r_{a}(i), r_{b}(i)$ and $r_{a b}(i)$, and the corresponding standard deviations. In nonsurvivors, the four panels clearly show a significant increase of each considered 
feature together with higher values for the standard deviations. Moreover, we have computed unpaired t-tests to assess whether the data in the two different classes are significantly different from each other. We have found $p$-values smaller than 0.01 for all the features in both the humeri, while five features out of six in the femurs result in a significance level of 0.05 , the sixth one showing a $p$-value of $\sim 0.09$.

\section{$>$ Remarks}

1. Although we are looking for variations in the values of the parameters characterizing the inner ellipses, considering the ratios (16)-(18) allows us to normalize and make them comparable within a population of subjects differing for age, sex and duration of the disease.

2. Relations (16) and (17) deal with ratios of linear quantities, while relation (18) is a second-order feature strictly related to the trabecular bone volume evaluation performed in [15].

\section{Discussion and conclusions}

This paper deals with the application of an HT-based approach to the recognition of the compact bone contours in biomedical images, as a tool to assess the impact of Advanced Chronic Lymphocytic Leukemia on the skeleton tissue. We have analyzed CT images of sixteen subjects, seven of which victims of ACLL, and for each appendicular bone we have recognized the inner and outer profiles using ellipses.

As proved in a previous work, ACLL erodes the compact bone from within the trabecular tissue [15]. As a consequence, no significant alterations are expected on the semi-axes values associated to the outer ellipses over the whole population. On the contrary, in the high-risk population alterations of the semi-axes values associated to the inner ellipses with respect to the corresponding values in control subjects are expected. Coherently, the ratios between the inner and outer values for each pair of semi-axes are expected to increase. We have then utilized the parameters characterizing the ellipses, in particular their ratios, to look for findings of bone alteration in the population affected by ACLL compared with the control subjects. To this aim, we have computed first-order and second-order features that all show significant variations in the values of the parameters characterizing the inner ellipses in presence of disease and then showing for the first time geometrical aspects of the erosion. The different behavior of the two populations, with particular regard to the humeri, is confirmed with a high level of significance by standard statistical tests and Kaplan-Meier analysis. From a quantitative point of view, after stratification according to median values of such features, patients cluster in two groups showing a two-years survival of $87.5 \%$ and of $25 \%$ according to whether their data are be- 
low and above the median, respectively. Further, a t-test analysis, to assess whether the class of control subjects plus the ACLL survivor and the class of ACLL victims are statistically different from each other, showed a significance level $p \leq 0.01$ for the features corresponding to the humeri and $0.01<p \leq 0.05$ in the femurs case. The method, used in [15], and based on the computational tool validated in [28], was able to provide a previously undisclosed imaging-based prognostic index. The classification outlined in the present paper mirrors and extends previous results, allowing to obtain a more reliable and flexible index to automatically analyze the skeletal composition. This method is able to overcome the main limitation of the approach used in [15], that is, the use of a rather arbitrary thresholding step for the recognition of the (highly informative) bone inner profile. We performed a comparison of both methods with a ground truth given by manual segmentation from expert users, and we have computed the percentage error committed by the two methods in the evaluation of the trabecular bone area with respect to the ground truth value. The result is that the HT-based method significantly outperforms the thresholding procedure, as shown in Table 3. In conclusion, the present study introduces an improved computational method to quantify skeletal alterations in hematologic malignancies. This new technique appears to be reproducible, operator-independent, and could eliminate a number of technical limitations of the formerly proposed active contours/thresholding method, such as the presence of focal interruptions or other disease-related structural alterations, thus allowing for a better characterization and prognostic assessment of hematologic patients. Furthermore, it allows the introduction of the image analysis concept in a patient population where a visual approach of neither morphological nor functional images was able to attain a diagnosis or to formulate a prognosis. Accordingly, further studies should be aimed to test this computational method in several scenarios of hematological or non-hematological-related skeletal alterations, in which the previous approach [28] presented specific limitations, related to the compact bone sampling method or to the inner profile recognition. The limitation of the present study is the relatively small number of subjects at disposal. The present project represents in fact a proof of concept, describing the potential of the application of the Hough transform in a model of leukemiaenvironment interaction. A rigorous application of the developed method to a large dataset of ACLL patients could assess the diagnostic potential of the features defined in this work and based on the application of the Hough transform to the recognition of bone contours in CT images. To this purpose, it is important to outline how the parameters occurring in the equations defining the curves of the families we deal with, could become concise features for the analysis of the results of the HT-based recognition procedure.

A preliminary version of the Matlab-based software implementing the procedure presented in this paper is fully described in [9] and freely available at the following URL: http://mida.dima.unige.it/g_software_htbone.html. 
Acknowledgements We acknowledge support from INDAM grant (intensive period on Computational Methods for Inverse Problems in Imaging). We wish to thank Gianmario Sambuceti, Head of the Nuclear Medicine Unit of the IRCCS San Martino - IST, Università degli Studi di Genova, and our colleague and friend Michele Piana for many helpful discussions. Special thanks should be given to Annalisa Perasso for her contribution to the data analysis of this project.

\section{References}

1. Aslan MS, Ali A, Rara H, et al. A novel 3D segmentation of vertebral bones from volumetric CT images using graph cuts. Advances in Visual Computing, Lect. Notes in Comput. Sc. 2009;5876:519-528.

2. Ballard DH. Generalizing the Hough transform to detect arbitrary shapes. Pattern Recogn. 1981;13:111-122.

3. Beltrametti MC, Campi C, Massone AM and Torrente M-L. Geometry of the Hough transforms with applications to synthetic data, arXiv:1904.02587 [cs.CV]

4. Beltrametti MC, Massone AM, Piana M. Hough transform of special classes of curves. SIAM J. Imaging Sci. 2013;6:391-412.

5. Bonci A, Leo T, Longhi S. A bayesian approach to the Hough transform for line detection. IEEE Trans. Syst. Man. Cybern. Syst.—Part A: Systems and Humans 2005;35:945-955.

6. Boykov Y, Funka-Lea G. Graph cuts and efficient N-D image segmentation. Int. J. Comput. Vision 2006;70:109-131.

7. Boykov Y, Jolly M-P. Interactive graph cuts for optimal boundary \& region segmentation of objects in N-D images. International Conference on Computer Vision 2001; I:105-112.

8. Campi C, Perasso A, Beltrametti MC, Massone AM, Sambuceti G and Piana M. Pattern recognition in medical imaging by means of the Hough transform of curves, Proc. of 8th International Symposium on Image and Signal Processing and Analysis (ISPA 2013), 280283.

9. Campi C, Perasso A, Beltrametti MC, Sambuceti G, Massone AM and Piana M. HT-BONE: A Graphical User Interface for the identification of bone profiles in CT images via extended Hough transform, Proc. SPIE 9784, Medical Imaging 2016: Image Processing, 978423.

10. Canny J. A computational approach to edge detection. IEEE Trans. on Pattern Anal. Mach. Intell. 1986;8:679-698.

11. Caselles V, Kimmel R, Sapiro G. Geodesic active contours. Int. J. of Comput. Vision 1997;22:61-79.

12. Chan TF, Vese LA. Active contours without edges. IEEE Trans. Image Process. 2001;10:266277.

13. Ciliberto C. Algebra Lineare. Programma di Matematica, Fisica, Elettronica. Bollati Boringhieri; 1994

14. Duda RO, Hart PE. Use of the Hough transformation to detect lines and curves in pictures. Commun. ACM. 1972;15:11-15.

15. Fiz F, Marini C, Piva R, et al. Adult advanced chronic lymphocytic leukemia: Computational analysis of whole-body CT documents a bone structure alteration. Radiology 2014;271:805813.

16. Haralick RM, Shapiro LG. Computer and Robot Vision, 1st edition. Boston, MA: AddisonWesley Longman Publishing Co Inc; 1992.

17. Hough PVC. Method and means for recognizing complex patterns. U.S. Patent 3069654; 1962.

18. Hudson HM, Larkin RS. Accelerated image reconstruction using ordered subsets of projection data. IEEE Trans. Med. Imaging 1994;13:601-609.

19. Kaplan EL, Meier P. Nonparametric estimation from incomplete observations. J. Am. Stat. Assoc. 1958;53:457-481. 
20. Malladi R, Sethian JA, Vemuri BC. Shape modeling with front propagation: a level set approach. IEEE Trans. Pattern Anal. Mach. Intell. 1995;17:158-175.

21. Massone AM, Perasso A, Campi C, et al. Profile detection in medical and astronomical images by means of the Hough transform of special classes of curves. J. Math. Imaging Vis. 2015;51:296-310.

22. Paragios N, Mellina-Gottardo O, Ramesh V. Gradient vector flow fast geometric active contours. IEEE Trans. Pattern Anal. Mach. Intell. 2004;26:402-407.

23. Philip KP, Dove EL, McPherson DD, et al. The Fuzzy Hough Transform-Feature Extraction in Medical Images. IEEE Trans. Med. Imaging 1994;13:235-240.

24. Ricca G, Beltrametti MC, Massone AM. Piecewise recognition of bone skeleton profiles via an iterative Hough transform approach without re-voting. Medical Imaging 2015: Image Processing, Proc. of SPIE. 2015;9413:94132M:1-8.

25. Ricca G, Beltrametti MC, Massone AM. Detecting curves of symmetry in images via Hough transform. Math. Comput. Sci., Special Issue on Geometric Computation 2016;10:179-205.

26. Robbiano L. Hyperplane Sections, Gröbner bases, and Hough transforms. J. Pure Appl. Algebra 2015;219:2434-2448.

27. Rosset A, Spadola L, Ratib O. OsiriX: An open-source software for navigating in multidimensional DICOM images. J. Digit. Imaging 2004;17:205-216.

28. Sambuceti G, Brignone M, Marini C, et al. Estimating the whole bone-marrow asset in humans by a computational approach to integrated PET/CT imaging. Eur. J. Nucl. Med. Mol. Imaging 2012;39:1326-1338.

29. Torrente M-L, Beltrametti MC. Almost-vanishing polynomials and an application to the Hough transform. J. Algebra Appl. 2014;13:39pp.

30. Truc PTH, Kim YH, Lee YK, et al. Evaluation of active contour-based techniques toward bone segmentation from CT images. World Congress on Medical Physics and Biomedical Engineering 2006, IFMBE Proceedings, Springer, Berlin Heidelberg. 2007;14:3121-3125.

31. Vese LA, Chan TF. A multiphase level set framework for image segmentation using the Mumford and Shah model. Int. J. Comput. Vision 2002;50:271-293.

32. Xu C, Prince JL. Snakes, shapes, and gradient vector flow. IEEE Trans. Image Process. 1998;7:359-369.

33. Yezzi A, Kichenassamy S, Kumar A, et al. A geometric snake model for segmentation of medical imagery. IEEE Trans. Med. Imaging 1997;16:199-209.

34. Zhang J, Yan $\mathrm{CH}$, Chui CK, et al. Fast segmentation of bone in $\mathrm{CT}$ images using 3D adaptive thresholding. Comput. Biol. Med. 2010;40:231-236. 